\title{
Shifted Chebyshev reproducing kernel method for flow of an electrically conducting nanofluid over an impermeable stretching cylinder problem
}

\author{
Mohammadreza Foroutan ${ }^{1}$, Mir Sajjad Hashemi², and Fatemeh Habibi ${ }^{2}$ \\ ${ }^{1}$ Payame Noor University \\ ${ }^{2}$ University of Bonab
}

June 5, 2020

\begin{abstract}
In this study a reproducing kernel Hilbert space method with Chebyshev function is proposed for approximating solutions of a nonlinear system of ordinary differential equations under multi-point boundary conditions. Based on reproducing kernel theory, reproducing kernel functions with a polynomial form will be erected in the reproducing kernel spaces spanned by the shifted Chebyshev polynomials. Convergence analysis of the proposed technique is theoretically investigated. This approach is successfully used for solving a system of ordinary differential equations with multi-point boundary conditions arising in flow of an electrically conducting nanofluid over an impermeable stretching cylinder.
\end{abstract}

\section{Hosted file}

FHH.pdf available at https://authorea.com/users/330463/articles/457206-shifted-chebyshevreproducing-kernel-method-for-flow-of-an-electrically-conducting-nanofluid-over-animpermeable-stretching-cylinder-problem 
figures/Fig0/Fig0-eps-converted-to.pdf 
figures/Fig1/Fig1-eps-converted-to.pdf 
figures/Fig2/Fig2-eps-converted-to.pdf 
figures/Fig3/Fig3-eps-converted-to.pdf 
figures/Fig4/Fig4-eps-converted-to.pdf 
figures/Fig5/Fig5-eps-converted-to.pdf 
figures/Fig6/Fig6-eps-converted-to.pdf 
figures/Fig7/Fig7-eps-converted-to.pdf 
figures/Fig8/Fig8-eps-converted-to.pdf 
figures/Fig9/Fig9-eps-converted-to.pdf 
figures/Fig10/Fig10-eps-converted-to.pdf 
figures/Fig11/Fig11-eps-converted-to.pdf 
figures/Fig12/Fig12-eps-converted-to.pdf 
figures/res $1 \mathrm{a} / \mathrm{res} 1 \mathrm{a}-\mathrm{eps}$-converted-to.pdf 
figures/res $1 b / r e s 1 b$-eps-converted-to.pdf 
figures/res1c/res1c-eps-converted-to.pdf 
figures/res $2 a / r e s 2 a-e p s-c o n v e r t e d-t o . p d f$ 
figures/res $2 b / r e s 2 b$-eps-converted-to.pdf 
figures/res $2 c / r e s 2 c-e p s-c o n v e r t e d-t o . p d f$ 
figures/res3a/res3a-eps-converted-to.pdf 
figures/res3b/res3b-eps-converted-to.pdf 
figures/res3c/res3c-eps-converted-to.pdf 